Ingeniare. Revista chilena de ingeniería, vol. $15 \mathrm{~N}^{\mathrm{o}} 1,2007, \mathrm{pp} .55-64$

\title{
SIMULACIÓN MATEMÁTICA DEL PROCESO DE SECADO DE LA GRACILARIA CHILENA (GRACILARIA CHILENSIS)
}

\section{MATHEMATICAL SIMULATION OF DRYING PROCESS OF CHILEAN GRACILARIA (GRACILARIA CHILENSIS)}

\author{
Antonio Vega Gálvez ${ }^{1} \quad$ Cristian Tello Ireland $^{1} \quad$ Roberto Lemus Mondaca $^{1}$ \\ Recibido 8 de agosto de 2006, aceptado 7 de noviembre de 2006 \\ Received: August 8, 2006 Accepted: November 7, 2006
}

\begin{abstract}
RESUMEN
El objetivo de este trabajo es estudiar y modelar la cinética de secado por aire caliente del alga Gracilaria (Gracilaria chilensis) utilizando un secador convectivo diseñado y construido en la Facultad de Ingeniería de la Universidad de La Serena a cinco temperaturas de bulbo seco $\left(30,40,50,60\right.$ y $\left.70^{\circ} \mathrm{C}\right)$ y velocidad de aire de $2.0 \pm 0.2 \mathrm{~m} \cdot \mathrm{s}^{-1}$. Para el modelado matemático se utilizan tres modelos empíricos (Newton, Henderson-Pabis \& Page). Durante el experimento se observa solamente el periodo de velocidad decreciente, por lo que se utiliza la ecuación de la segunda Ley de Fick para el cálculo de la difusividad efectiva de agua. El proceso de secado presenta humedades finales entre $0.096 \mathrm{~g}$ agua $/ \mathrm{g} \mathrm{m} . \mathrm{s} \mathrm{y} 0.061 \mathrm{~g}$ agua $/ \mathrm{g} \mathrm{m} . \mathrm{s}$ para $30^{\circ} \mathrm{C}$ y $70^{\circ} \mathrm{C}$, respectivamente. Tanto la difusividad como los parámetros cinéticos $\mathrm{k}_{1}, \mathrm{k}_{2} \mathrm{y} \mathrm{k}_{3}$ de los modelos propuestos presentan dependencia con la temperatura y al evaluarlos con la ecuación de Arrhenius se obtienen energías de activación de 39.92, $33.85,33.49$ y $33.83 \mathrm{~kJ} \cdot \mathrm{mol}^{-1}$, respectivamente. De acuerdo a los análisis estadísticos que se utilizan $\left(\mathrm{r}^{2}\right.$, SSE, RMSE y $\left.\chi^{2}\right)$, el modelo de Page muestra la mejor calidad de ajuste sobre los datos experimentales, otorgando así una buena herramienta para el modelado de la cinética de secado industrial de la Gracilaria chilensis y el cálculo del tiempo de secado a diferentes temperaturas, con el fin de alcanzar un contenido de humedad comercial aceptable internacionalmente.
\end{abstract}

Palabras clave: Gracilaria, secado, difusividad, Arrhenius, Page.

ABSTRACT

The aim of this research is to study and to model the hot air drying kinetics of Gracilaria algae (Gracilaria chilensis), using a convective drier - designed and built at the Faculty of Engineering of Universidad de La Serena-at five dry bulb temperatures $\left(30,40,50,60\right.$ and $\left.70^{\circ} \mathrm{C}\right)$ and an air velocity of $2.0 \pm 0.2 \mathrm{~m} \cdot \mathrm{s}^{-1}$. Three empirical models are used for the mathematic modeling (Newton, Henderson-Pabis \& Page). During the experiment, only a falling rate period is observed, hence the Fick's second law equation is used to estimate the effective water diffusivity. The drying process shows final moisture contents between $0.096 \mathrm{~g}$ water $/ \mathrm{g}$ d.b. and $0.061 \mathrm{~g}$ water $/ \mathrm{g}$ d.b. for $30^{\circ} \mathrm{C}$ and $70^{\circ} \mathrm{C}$, respectively. Both diffusivity and kinetic parameters $k_{1}$, $k_{2}$ and $k_{3}$ of the proposed models show dependence on temperature, and when being evaluated by the Arrhenius equation, activation energies of 39.92, 33.85, 33.49 and $33.83 \mathrm{~kJ} \cdot \mathrm{mol}^{-1}$ are obtained, respectively. According to the statistical analyses used $\left(r^{2}\right.$, SSE, RMSE and $\left.\chi^{2}\right)$, the Page model shows the best fit quality on experimental data, thus being considered an excellent tool for the kinetic modeling of industrial drying processing of Gracilaria chilensis and the estimation of drying time at different temperatures, in order to achieve a commercial moisture content to be acceptable abroad.

Keywords: Gracilaria, drying, diffusivity, Arrhenius, Page.

\section{INTRODUCCIÓN}

La Gracilaria (Gracilaria chilensis) pertenece a las algas del género Gracilaria y orden Gracilariales, es comúnmente llamada "pelillo", y su distribución geográfica en Chile es entre la región de Coquimbo y la isla de Chiloé (IV a $\mathrm{X}$ región), teniendo como hábitat bahías protegidas con fondos arenosos y areno-fangosos de aguas marinas poco profundas como estuarios, y en algunos casos adheridos a sustratos duros [1]. En Chile el rendimiento anual del cultivo de Gracilaria puede llegar a 126.000 toneladas, cerca del $60 \%$ proviene de cultivos en praderas [2]. Las exportaciones entre enero y noviembre del 2004 alcanzaron las 27.000 toneladas de alga seca, lo que representó el $21 \%$ de las exportaciones de productos pesqueros según línea de elaboración [3].

1 Departamento de Ingeniería en Alimentos. Universidad de La Serena. Casilla 599. La Serena, Chile, e-mail: avegag@userena.cl 
El alto valor comercial de la Gracilaria deshidratada, junto al Gelidium y Gigartina (otras algas marinas), se debe a que son las principales fuentes para la extracción del agar, representando éstas casi el $95 \%$ de la producción mundial [3]. El agar tiene un amplio uso en áreas tales como biotecnología, microbiología, medicina, química, farmacia y en el campo de la ciencia y tecnología de los alimentos. En esta última industria, su utilidad se debe a que actúa como un agente gelificante, ya que presenta la capacidad de absorber y retener humedad según sea su calidad, la que depende de la materia prima (tipo de alga), de los métodos de extracción y condiciones de secado. Algunos de los alimentos que contienen agar en su formulación se encuentran las cecinas, jaleas, yogures, conservas, helados, etc. [4].

Actualmente el secado de la Gracilaria se realiza en condiciones ambientales (secado al sol), sin embargo, una de las operaciones unitarias más utilizada en la industria de los alimentos es el secado por aire caliente, el cual es un proceso simultáneo de transferencia de calor y masa, acompañado por un cambio de fase [5]. El secado como método de preservación industrial es ampliamente usado para disminuir la actividad de agua de los alimentos, por lo que se minimizan las reacciones bioquímicas de degradación en el almacenamiento a temperatura ambiente [6]. No obstante, este proceso depende de varios factores internos, por ejemplo, el espesor y geometría del producto, y de ciertas condiciones del aire de secado, tales como la temperatura, humedad relativa y velocidad [7].

Para controlar y optimizar el proceso del secado se hace necesaria la utilización de ecuaciones matemáticas para la simulación de la cinética de transferencia de materia (agua) ocurrida durante esta operación unitaria, en función de las condiciones de secado utilizadas. La mayoría de estos modelos son empíricos, pero de igual forma derivan del modelo difusional de la segunda ley de Fick para diferentes geometrías [8]. El modelo difusional de Fick considera que el transporte de agua desde el interior del sólido hacia la superficie del mismo tiene lugar principalmente por difusión, calculándose de este modo un coeficiente de difusión efectivo [9].

Las isotermas de sorción de humedad en los alimentos muestran la relación que existe entre el contenido de humedad de equilibrio del alimento y la actividad de agua del mismo $\left(\mathrm{a}_{\mathrm{w}}\right)$ a una temperatura y presión constante [10]. Es por esto que para iniciar el estudio de secado por aire caliente del producto es necesario obtener la humedad de equilibrio, la cual se determina por medio de la isoterma de desorción del producto fresco [11].
El objetivo de este trabajo fue estudiar y simular mediante ecuaciones empíricas la cinética de secado por aire caliente de la Gracilaria chilena (Gracilaria chilensis) a cinco temperaturas de bulbo seco, además de obtener el coeficiente difusional efectivo de agua, con el fin de poder estimar el tiempo de secado para obtener un alga seca de calidad comercial.

\section{MATERIALES Y MÉTODOS}

La Gracilaria fue extraída de la bahía de La Herradura, ciudad de Coquimbo, de la concesión marítima perteneciente a la empresa Sea Farmers. Los lotes obtenidos se lavaron con agua fría para eliminar restos físicos como arena, conchillas y otras algas pequeñas. La determinación de la humedad inicial se realizó a través de la metodología gravimétrica [12], utilizando una estufa a vacío (Gallenkamp) y una balanza analítica, de precisión $0.0001 \mathrm{~g}$ (CHYO Jex-120).

La isoterma de desorción de la gracilaria fue obtenida para tres temperaturas de trabajo 5,25 y $40^{\circ} \mathrm{C}$. La metodología para la determinación de la isoterma de desorción es la recomendada por el Proyecto Europeo COST 90 [13]. Esta metodología consiste en tomar una masa conocida de muestra (fresca), la que se deja equilibrar con la atmósfera producida por una disolución saturada de sal de humedad relativa conocida dentro de un recipiente herméticamente cerrado (tabla 1).

Tabla 1. Sales utilizadas y su correspondiente humedad relativa a las tres temperaturas.

\begin{tabular}{|l|c|c|c|}
\hline \multirow{2}{*}{ Sales } & \multicolumn{3}{|c|}{$\% \mathrm{HR}$} \\
\cline { 2 - 4 } & $5^{\circ} \mathrm{C}$ & $25^{\circ} \mathrm{C}$ & $40^{\circ} \mathrm{C}$ \\
\hline Cloruro de litio & 12.89 & 11.42 & 10.54 \\
\hline Acetato de potasio & 29.13 & 23.67 & 20.61 \\
\hline Cloruro de magnesio & 35.37 & 32.87 & 31.31 \\
\hline Carbonato de potasio & 45.90 & 44.32 & 43.30 \\
\hline Nitrato de magnesio & 58.39 & 53.58 & 50.60 \\
\hline Bromuro de sodio & 63.78 & 57.32 & 53.39 \\
\hline Ioduro de potasio & 73.28 & 68.90 & 66.12 \\
\hline Cloruro de sodio & 80.49 & 76.17 & 73.42 \\
\hline Cloruro de potasio & 93.38 & 85.46 & 80.56 \\
\hline Nitrato de potasio & 97.88 & 92.28 & 88.72 \\
\hline Sulfato de potasio & 98.87 & 97.56 & 96.70 \\
\hline
\end{tabular}

Los datos experimentales de la isoterma de desorción se modelaron con la ecuación propuesta por Guggenheim, Anderson y de Boer, comúnmente llamada GAB (ecuación 1), la cual es de amplio uso en alimentos y productos vegetales [15]. 
Desde ese instante se controló el peso de las muestras cada 15 días hasta llegar a peso constante (condición de equilibrio). A los recipientes que contenían disoluciones con humedad relativa superior al $75 \%$ se les adjuntó una placa Petri con Thymol, para evitar el desarrollo de hongos [14]. En el momento que las muestras llegaron a peso constante, se determinó el contenido de humedad por medio de la metodología gravimétrica [12].

$$
X_{w e}=\frac{X_{m} \cdot C \cdot k \cdot a_{w}}{\left(1-k \cdot a_{w}\right) \cdot\left(1+(C-1) \cdot k \cdot a_{w}\right)}
$$

El criterio para evaluar la calidad de ajuste de este modelo fue el coeficiente de regresión lineal $\left(\mathrm{r}^{2}\right)$ y el error porcentual medio relativo (\%E) (ecuación 2$)$.

$$
\% E=\frac{100}{N} \cdot \sum_{i=1}^{N} \frac{\left|X_{e i}-X_{c i}\right|}{X_{e i}}
$$

El proceso de deshidratación se llevó a cabo en un secador convectivo de bandejas, diseñado y construido por el Depto. Ingeniería en Alimentos de la Universidad de La Serena, el que posee un ventilador, un panel de control que monitorea la velocidad y temperatura de aire de secado, que se calienta al pasar por un sistema calefactor que tiene resistencias eléctricas (figura 1).

Para el estudio de la cinética de secado se utilizaron 5 diferentes temperaturas del bulbo seco, 30, 40, 50, 60 y $70^{\circ} \mathrm{C}$, con una velocidad de aire constante de $2.0 \pm$ $0.2 \mathrm{~m} \cdot \mathrm{s}^{-1}$. Todas las experiencias de secado se realizaron en triplicado, considerando una masa de muestra de $100 \pm 2 \mathrm{~g}$. La masa se registró con una balanza analítica (Ohaus SP402) de precisión $0.01 \mathrm{~g}$ a intervalos de tiempo definido por un sistema interfase (Ohaus RS232) comunicado desde la balanza al computador. Las experiencias finalizaron cuando se alcanzó peso constante (equilibrio). Luego las muestras fueron retiradas y envasadas en bolsas de polipropileno.

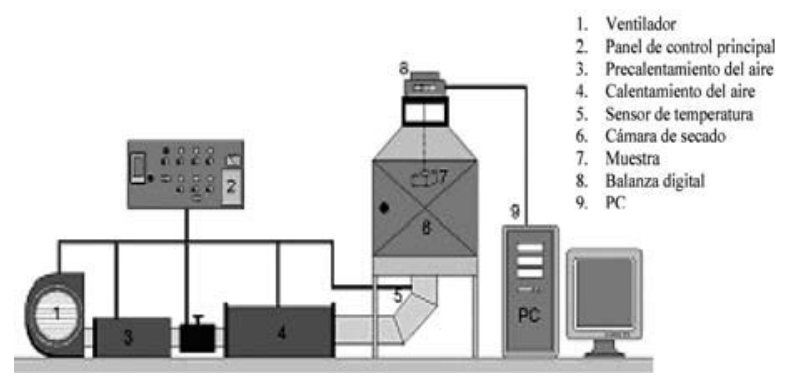

Figura 1. Secador Convectivo del Departamento de Ingeniería en Alimentos, Universidad de La Serena, Chile.
La difusividad efectiva de agua $\left(\mathrm{D}_{\text {we }}\right)$ se determinó por el método que tradicionalmente ha sido utilizado en el estudio de la transferencia de masa en estado no estacionario para el secado de productos vegetales, esto es, la ecuación integrada de la segunda ley de Fick [14]. En esta ocasión se consideraron tiempos largos de proceso, y ya que la Gracilaria es de forma cilíndrica se utilizó una geometría cilíndrica infinita de radio uniforme $0.01 \mathrm{~m}$. La ecuación 3 representa el primer término del desarrollo de la serie [16].

$$
M R=\frac{X_{w t}-X_{w e}}{X_{w o}-X_{w e}}=\frac{4}{5.783} \cdot \exp \left(\frac{-5.783 \cdot D_{w e} \cdot t}{\text { radio }^{2}}\right)
$$

Para observar si existe influencia de la temperatura de secado sobre la difusividad del agua desde el interior del producto hacia la superficie del mismo, se aplicó la ecuación de Arrhenius (ecuación 4) con la cual se puede obtener la energía de activación $\left(\mathrm{E}_{\mathrm{a}}\right)$, que representa la energía para iniciar la reacción de difusión [10].

$$
\left.D_{w e}=D_{o} \cdot e^{\left[-E_{a} / R T\right.}\right]
$$

Donde $\mathrm{D}_{\mathrm{we}}$ es la difusividad efectiva de agua $\left(\mathrm{m}^{2} \cdot \mathrm{s}^{-1}\right)$; $\mathrm{D}_{\mathrm{o}}$ es el factor de Arrhenius $\left(\mathrm{m}^{2} \cdot \mathrm{s}^{-1}\right) ; \mathrm{E}_{\mathrm{a}}$ es la energía de activación $\left(\mathrm{kJ} \cdot \mathrm{mol}^{-1}\right)$; $\mathrm{T}$ es la temperatura absoluta de secado $(\mathrm{K})$ y $\mathrm{R}$ es la constante universal de los gases $\left(8.314 \mathrm{~J} \mathrm{~mol}^{-1} \mathrm{~K}^{-1}\right)$.

De los tres modelos utilizados en esta investigación, el modelo más simple es el conocido como el modelo exponencial o modelo de Newton (ecuación 5), luego se presenta el modelo de Page (ecuación 6) que presenta una modificación empírica del anterior introduciendo el exponente " $n$ ", al igual que el modelo de Henderson-Pabis (ecuación 7) que considera otro parámetro " $a$ " $[6,17]$.

$$
\begin{array}{ll}
\begin{array}{l}
\text { Modelo de } \\
\text { Newton }
\end{array} & \frac{X_{w t}-X_{w e}}{X_{w o}-X_{w e}}=\exp \left(-k_{1} t\right) \\
\text { Modelo de } & \frac{X_{w t}-X_{w e}}{X_{w o}-X_{w e}}=\exp \left(-k_{2} t^{n}\right) \\
\text { Page } & \\
\text { Modelo } & \frac{X_{w t}-X_{w e}}{X_{w o}-X_{w e}}=a \cdot \exp \left(-k_{3} t\right)
\end{array}
$$

Estos modelos empíricos derivan de una relación directa entre el contenido de humedad promedio y el tiempo de secado, además de estar fuertemente relacionados con el modelo difusional de la segunda ley de Fick [8]. 
Para todos los modelos propuestos se evaluó la calidad de ajuste sobre los datos experimentales con el coeficiente de regresión lineal $\left(\mathrm{r}^{2}\right)$, la suma de los errores cuadrados (SSE) (ecuación 8), la raíz media de los errores cuadrados (ecuación 9) y el parámetro Chi- cuadrado (ecuación 10) [17].

$$
\begin{aligned}
S S E & =\frac{1}{N} \sum_{i=1}^{N}\left(M R_{e i}-M R_{c i}\right)^{2} \\
R M S E & =\left[\frac{1}{N} \sum_{i=1}^{N}\left(M R_{c i}-M R_{e i}\right)^{2}\right]^{1 / 2} \\
\chi^{2} & =\frac{\sum_{i=1}^{N}\left(M R_{e i}-M R_{c i}\right)^{2}}{N-z}
\end{aligned}
$$

\section{RESULTADOS Y DISCUSIÓN}

La humedad inicial de las muestras de Gracilaria fue de $6.25 \pm 0.40 \mathrm{~g}$ agua $/ \mathrm{g}$ m.s. En la figura 2 se puede apreciar que se obtuvieron isotermas del tipo II, de forma sigmoidea, de las cinco establecidas por Van der Waals, las cuales son las más frecuentes en alimentos como frutas y verduras [15]; además se aprecia en la misma figura 2, la buena calidad de ajuste de la ecuación de GAB sobre los datos experimentales para cada isoterma de desorción.

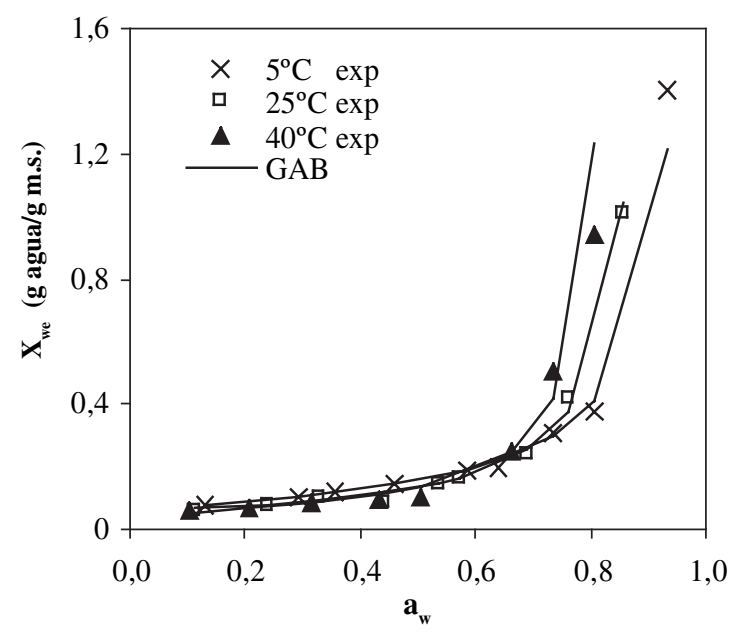

Figura 2. Isotermas de desorción de la Gracilaria modeladas con la ecuación de GAB.

La tabla 2 muestra los valores obtenidos de los parámetros de la ecuación de GAB, donde el valor de la humedad de la monocapa $\left(\mathrm{X}_{\mathrm{m}}\right)$ muestra una tendencia a disminuir a medida que aumenta la temperatura, esto se puede deber a la menor disponibilidad de los sitios activos (o enlaces de hidrógeno) en los polímeros de la Gracilaria para la unión con el agua [4].

Además, en la misma tabla se observa un excelente coeficiente de regresión lineal, mayor a 0.98 y un error porcentual medio relativo menor a $10 \%$ para las tres temperaturas utilizadas, confirmando que la ecuación de GAB es un buen modelo para predecir la humedad de equilibrio de la Gracilaria chilena a un amplio rango de humedad relativa $(0.10-0.98)$.

Como se comentó anteriormente, las muestras de Gracilaria fueron deshidratadas por aire caliente a diferentes temperaturas de bulbo seco $\left(30,40,50,60\right.$ y $\left.70^{\circ} \mathrm{C}\right)$. La tabla 3 muestra las humedades de equilibrio calculadas a partir de la isoterma de desorción a $40^{\circ} \mathrm{C}$, para cada temperatura de trabajo.

Tabla 2. Parámetros de la ecuación de GAB para las tres temperaturas de trabajo, además de su $\mathrm{r}^{2} \mathrm{y} \% \mathrm{E}$.

\begin{tabular}{|c|c|c|c|}
\hline Parámetros de GAB & $5^{\circ} \mathrm{C}$ & $25^{\circ} \mathrm{C}$ & $40^{\circ} \mathrm{C}$ \\
\hline $\mathrm{X}_{\mathrm{m}}$ & 0.08 & 0.06 & 0.05 \\
\hline $\mathrm{C}$ & 29.36 & 88.90 & 54.78 \\
\hline$k$ & 1.00 & 1.10 & 1.19 \\
\hline $\mathrm{r}^{2}$ & 0.98 & 0.99 & 0.98 \\
\hline$\% \mathrm{E}$ & 5.44 & 7.26 & 9.96 \\
\hline
\end{tabular}

Tabla 3. Humedad de equilibrio de la Gracilaria para cada temperatura de secado.

\begin{tabular}{|c|rcc|ccc|}
\hline$T\left({ }^{\circ} \mathrm{C}\right)$ & \multicolumn{2}{|c|}{$\%$ HR equilibrio $(\%)$} & \multicolumn{3}{|c|}{$X_{\text {we }}$ (g agua $/ g$ m.s.) } \\
\hline 30 & 35.5 & \pm & 2.5 & 0.099 & \pm & 0.009 \\
\hline 40 & 23.0 & \pm & 1.8 & 0.080 & \pm & 0.007 \\
\hline 50 & 9.1 & \pm & 1.6 & 0.062 & \pm & 0.008 \\
\hline 60 & 4.1 & \pm & 1.3 & 0.052 & \pm & 0.007 \\
\hline 70 & 1.9 & \pm & 0.7 & 0.041 & \pm & 0.004 \\
\hline
\end{tabular}

La figura 3 muestra las curvas de secado de las muestras de Gracilaria, las que se ven afectadas al aumentar la temperatura de trabajo, lo que lleva a disminuir el tiempo de secado y lograr el tiempo necesario para alcanzar y superar la humedad comercial del 18\% [2]. Similares resultados obtuvieron otros autores como [7, 10, 18 y 19].

En la figura 4 se muestran las curvas de velocidad de secado para las cinco temperaturas de trabajo de la Gracilaria chilena, en ella se observa una clara dependencia con la temperatura de secado, aumentando la pendiente de las mismas a medida que aumenta esta variable física. Además se puede observar un marcado período de velocidad decreciente con una clara tendencia exponencial, validando la utilización de los modelos propuestos para simular todo el proceso de secado, como [9, 10, 20 y 21]. 
Vega et al.: Simulación matemática del proceso de secado de la gracilaria chilena (gracilaria chilensis)

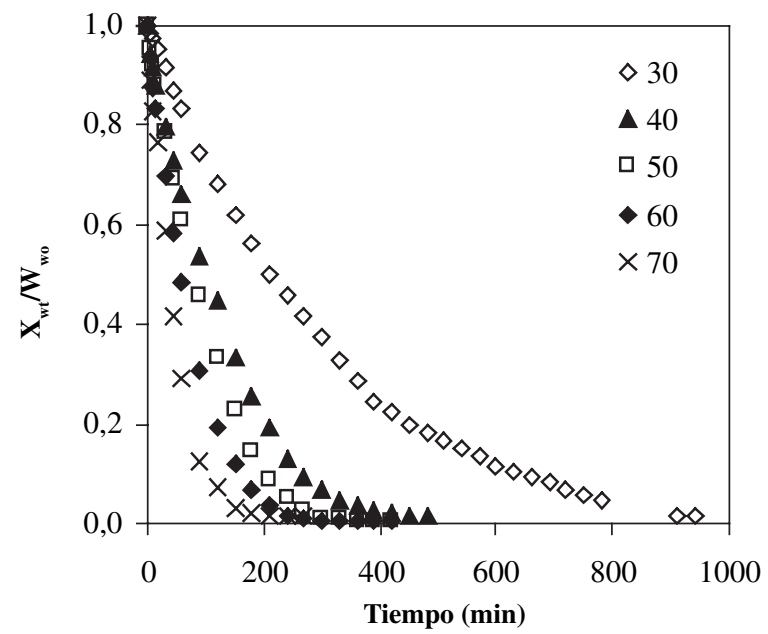

Figura 3. Curva de secado de Gracilaria chilena a diferentes temperaturas de secado $\left({ }^{\circ} \mathrm{C}\right)$.

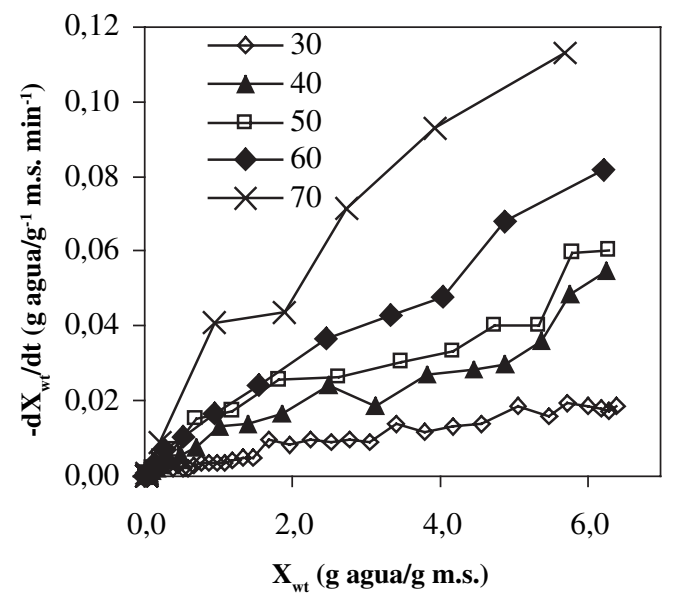

Figura 4. Curva de velocidad de secado de Gracilaria a diferentes temperaturas de secado $\left({ }^{\circ} \mathrm{C}\right)$.

Tiempo (s)

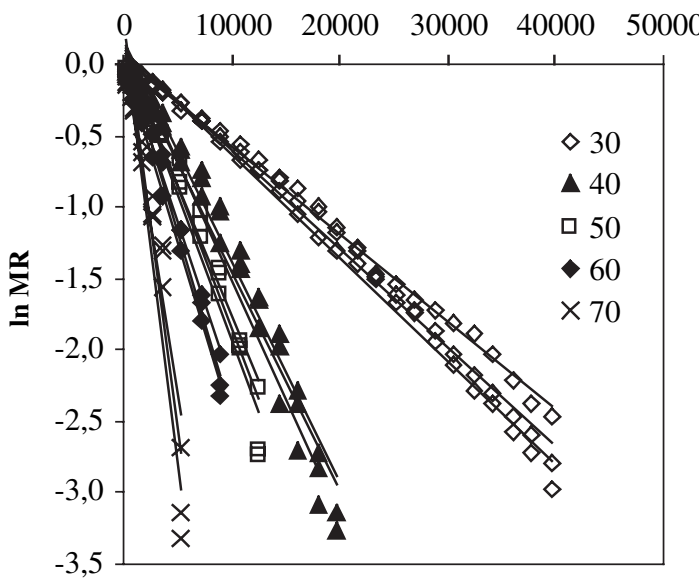

Figura 5. Logaritmo de MR (adimensional) versus el tiempo de secado (s) para cada curva de secado $\left({ }^{\circ} \mathrm{C}\right)$ en triplicado.
La ecuación 11 representa la linealización matemática de la ecuación 3, propuesta para una geometría cilíndrica infinita y tiempos largos de proceso.

$$
\ln M R=\ln \frac{4}{5.783}-\left(\frac{5.783 \cdot D_{w e} \cdot t}{\text { radio }^{2}}\right)
$$

Al graficar el logaritmo de la relación de humedad (ln $\mathrm{MR}$ ) en función del tiempo (segundos) se obtiene una línea recta (figura 5), de cuya pendiente se puede obtener el coeficiente difusional de agua $\left(\mathrm{D}_{\text {we }}\right)[17]$.

En la tabla 4 se presentan los valores promedios del coeficiente difusional de agua $\left(\mathrm{D}_{\text {we }}\right)$ para cada temperatura de trabajo, el cual se obtuvo a partir de las pendientes de las rectas de la figura 5 .

Tabla 4. Promedio y desviación estándar de las difusividades para cada temperatura.

\begin{tabular}{|c|rcc|}
\hline $\mathrm{T}\left({ }^{\circ} \mathrm{C}\right)$ & \multicolumn{3}{|c|}{ Difusividad $\left(\mathrm{x}^{-9} \mathrm{~m}^{2} \cdot \mathrm{s}^{-1}\right)$} \\
\hline 30 & 2.76 & \pm & 0.21 \\
\hline 40 & 6.39 & \pm & 0.33 \\
\hline 50 & 7.85 & \pm & 0.42 \\
\hline 60 & 9.83 & \pm & 0.59 \\
\hline 70 & 22.41 & \pm & 2.63 \\
\hline
\end{tabular}

En la tabla 4 se observa la clara dependencia de este parámetro con la temperatura, esto es a medida que aumentó la temperatura de secado aumentó la difusividad $\left(\mathrm{D}_{\text {we }}\right)$. Los valores obtenidos se encuentran dentro de lo establecido por [22] para la mayoría de los alimentos, esto es de $10^{-9}$ a $10^{-11} \mathrm{~m}^{2} \cdot \mathrm{s}^{-1}$. Y debido a la escasa fuente bibliográfica de secado de algas, ya sea al sol o por aire caliente, es que se tomaron como referencias otros estudios relacionados con frutas, vegetales y especias, tales como [10, 14, 17-20 y 23].

Como se observó en la figura 3, se presenta una clara tendencia exponencial de las curvas de secado, por lo que se sugiere la utilización de los modelos empíricos propuestos para la simulación del proceso de la cinética de secado por aire caliente de la Gracilaria chilena, siendo las ecuaciones de Newton, Page y Henderson-Pabis, los tres modelos utilizados en esta investigación.

En la tabla 5 se muestran los valores de los parámetros pertenecientes a los tres modelos empíricos utilizados para cada una de las temperaturas de secado. Observándose que los valores de los parámetros $\mathrm{k}_{1}, \mathrm{k}_{2} \mathrm{y} \mathrm{k}_{3}$ de estos modelos presentaron una clara tendencia a aumentar a medida que aumentó la temperatura del aire de secado. 
Del análisis de varianza (ANOVA) realizado por el programa Statgraphics Plus ${ }^{\circledR}$, versión 5.1, a las medias de las difusividades $\left(\mathrm{D}_{\text {we }}\right)$ para un nivel de confianza del $95 \%$, se obtuvo un valor $\mathrm{p}<0.05$, lo que significa que existe influencia significativa de la temperatura sobre este parámetro. Sin embargo, mediante un análisis estadístico aplicando el Multiple Range Test, del mismo programa, se detectaron 4 grupos homogéneos, 30, 40-50, 50-60 y $70^{\circ} \mathrm{C}$. Con el mismo test estadístico aplicado a las difusividades (ANOVA) se verificó la dependencia con la temperatura de los parámetros $\mathrm{k}_{1}, \mathrm{k}_{2} \mathrm{y} \mathrm{k}_{3}$, dando un valor $\mathrm{p}<0.05$, por lo que cada uno de estos parámetros presentaron diferencias estadísticamente significativas para las temperaturas de secado, además no presentaron grupos homogéneos con el análisis Multiple Range Test del mismo programa.

Para el coeficiente difusional se pudo obtener la energía de activación de $39.92 \mathrm{~kJ} \cdot \mathrm{mol}^{-1}$ con un factor de Arrhenius $\left(D_{\mathrm{o}}\right)$ de $2.30 \times 10^{-2} \mathrm{~m}^{2} \cdot \mathrm{s}^{-1}$. Valores similares a la energía de activación encontrada para la difusividad de la Gracilaria fueron obtenidos por [9-11, 19 y 21].

En las figuras 6 y 7 se puede observar, respectivamente, la dependencia del coeficiente difusional $\left(\mathrm{D}_{\mathrm{we}}\right)$ y de los parámetros cinéticos $\mathrm{k}_{1}, \mathrm{k}_{2} \mathrm{y} \mathrm{k}_{3}$ de los modelos empíricos propuestos con la temperatura de secado (K), por lo cual se aplicó la ecuación tipo Arrhenius para calcular la energía de activación (ecuación 12) [10].

$$
k_{i}=k_{o i} \cdot e^{\left[-E_{a} / R T\right]}
$$

Donde $\mathrm{k}_{\mathrm{i}}$ es el parámetro en estudio y $\mathrm{k}_{\mathrm{oi}}$ es el factor de Arrhenius. (i=1, 2, 3).

De acuerdo a lo obtenido a partir de la figura 7, los parámetros $\mathrm{k}_{1}, \mathrm{k}_{2} \mathrm{y} \mathrm{k}_{3}$ presentaron valores de coeficientes de regresión lineal superiores a 0.95 , para cada parámetro, demostrándose la dependencia de éstos con la temperatura de secado, así como también la obtención de la energía de activación para las mismas de 33.85, 33.49 y 33.83 $\mathrm{kJ} \cdot \mathrm{mol}^{-1}$, respectivamente.

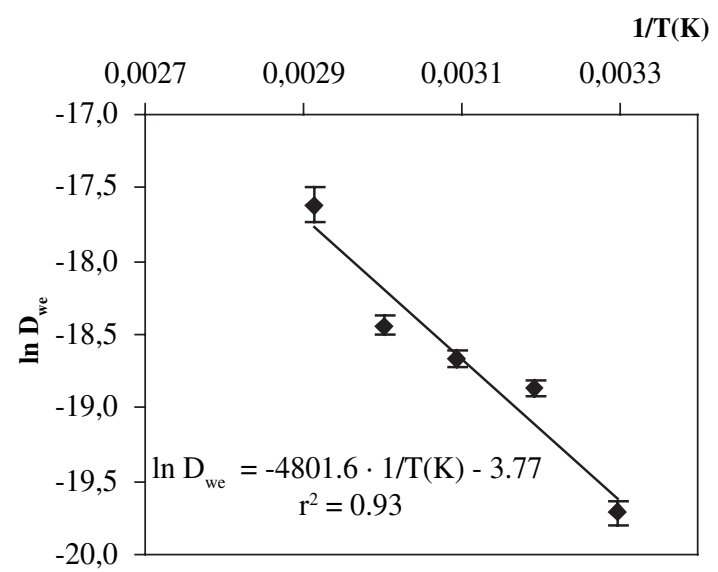

Figura 6. Análisis de regresión lineal para $\ln \mathrm{D}_{\mathrm{we}}$ versus la temperatura de secado (K).

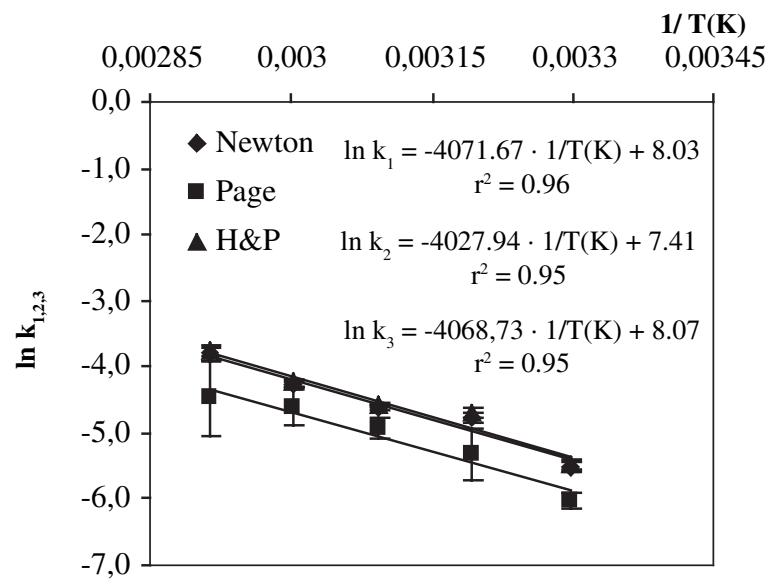

Figura 7. Ecuación de regresión lineal para los parámetros $\mathrm{k}_{\mathrm{i}}(\mathrm{i}=1,2,3)$.

Tabla 5. Valores de los parámetros cinéticos de cada modelo por curva de secado.

\begin{tabular}{|c|c|c|c|c|c|c|c|c|c|c|c|c|c|c|c|}
\hline \multirow{3}{*}{$\frac{\mathrm{T}\left({ }^{\circ} \mathrm{C}\right)}{30}$} & \multirow{2}{*}{\multicolumn{3}{|c|}{$\frac{\text { Newton }}{\mathrm{k}_{1} \times 10^{-3}\left(^{*}\right)}$}} & \multicolumn{6}{|c|}{ Page } & \multicolumn{6}{|c|}{ Henderson-Pabis } \\
\hline & & & & \multicolumn{3}{|c|}{$n$} & \multicolumn{3}{|c|}{$\mathrm{k}_{2} \times 10^{-3}(*)$} & \multicolumn{3}{|r|}{-} & \multicolumn{3}{|c|}{$\mathrm{k}_{3} \times 10^{-3}\left(^{*}\right)$} \\
\hline & 4.03 & \pm & 0.31 & 1.08 & \pm & 0.02 & 2.40 & \pm & 0.26 & 1.06 & \pm & 0.01 & 4.20 & \pm & 0.36 \\
\hline 40 & 8.57 & \pm & 0.60 & 1.07 & \pm & 0.03 & 5.13 & \pm & 0.20 & 1.05 & \pm & 0.05 & 9.07 & \pm & 0.74 \\
\hline 50 & 10.00 & \pm & 0.36 & 1.06 & \pm & 0.03 & 7.27 & \pm & 0.11 & 1.06 & \pm & 0.01 & 10.47 & \pm & 0.25 \\
\hline 60 & 14.00 & \pm & 0.85 & 1.02 & \pm & 0.03 & 10.20 & \pm & 0.27 & 1.05 & \pm & 0.04 & 14.47 & \pm & 0.84 \\
\hline 70 & 22.23 & \pm & 2.05 & 1.04 & \pm & 0.02 & 12.57 & \pm & 0.62 & 1.03 & \pm & 0.03 & 23.37 & \pm & 2.04 \\
\hline
\end{tabular}

$(*) \min ^{-1}$ 
Debido a la similitud de los valores de energía de activación entre el coeficiente difusional $\left(\mathrm{E}_{\mathrm{a}} 39.92 \mathrm{~kJ} \cdot \mathrm{mol}^{-1}\right)$ y los parámetros $\mathrm{k}_{1}, \mathrm{k}_{2} \mathrm{y} \mathrm{k}_{3}$, es que estos últimos podrían ser considerados como pseudodifusividades [11].

El parámetro $n$ del modelo de Page y el parámetro $a$ del modelo de Henderson-Pabis, con un valor promedio de $1.11 \pm 0.05$ y $1.05 \pm 0.03$, respectivamente, no presentaron dependencia con la temperatura, considerándose como parámetros constantes [24]. En otras investigaciones el parámetro $n$ del modelo de Page también se mantuvo constante, con valores entre 1.06 y 2.05 [10]. [25] proponen que valores del parámetro $n$ de Page dependen del tipo de producto que se va a deshidratar y no de la temperatura de secado. Otros estudios concluyen que $n$ está en función de la velocidad del aire y la presencia de piel [24].

Para el caso del parámetro $\mathrm{k}_{2}$ del modelo de Page, éste aumenta su valor al aumentar la temperatura del aire de secado, lo cual concuerda con otros estudios como [10, 19 y 26], esto puede deberse a que como lo mencionan [27] el parámetro $\mathrm{k}_{2}$ está en función de la temperatura del aire de secado y el contenido de humedad inicial.

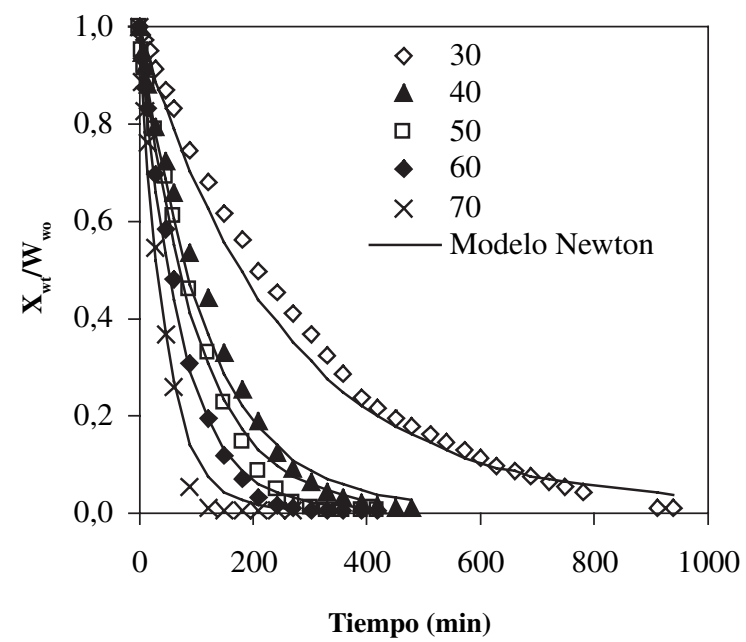

Figura 8. Curva de secado experimental y calculada con el modelo de Newton para las diferentes temperaturas de secado $\left({ }^{\circ} \mathrm{C}\right)$.
Las figuras 8, 9 y 10 muestran las curvas de secado para los valores experimentales y calculados a partir de los modelos de Newton, Page y Henderson-Pabis, respectivamente, representados por el cuociente entre la humedad a cada tiempo y la humedad inicial (adimensional) versus el tiempo de secado (min.).

La tabla 6 muestra los valores promedios y desviaciones estándares de los estadísticos propuestos para las cinco curvas de secado, con el fin de evaluar la calidad de ajuste de los modelos utilizados sobre los datos experimentales $\left(r^{2}\right.$, SSE, RMSE, $\left.\chi^{2}\right)$. Como buen ajuste se consideran los valores de SSE, RMSE y $\chi^{2}$ más cercanos a cero, junto con los valores más altos o que tiendan a uno para $\mathrm{r}^{2}[6,8,11]$.

Tabla 6. Promedio de las pruebas estadísticas para el modelo de Newton, Page y Henderson-Pabis.

\begin{tabular}{|c|c|c|c|}
\hline Estadístico & Newton & Page & H-P \\
\hline $\mathrm{r}^{2}$ & $0.98 \pm 0.01$ & $0.99 \pm 0.01$ & $0.99 \pm 0.01$ \\
\hline $\mathrm{SSE}$ & $0.0011 \pm 0.004$ & $0.0008 \pm 0.003$ & $0.0013 \pm 0.004$ \\
\hline $\mathrm{RMSE}$ & $0.0325 \pm 0.0060$ & $0.0269 \pm 0.0061$ & $0.0358 \pm 0.0061$ \\
\hline$\chi^{2}$ & $0.0012 \pm 0.0004$ & $0.0008 \pm 0.0004$ & $0.0015 \pm 0.0005$ \\
\hline
\end{tabular}

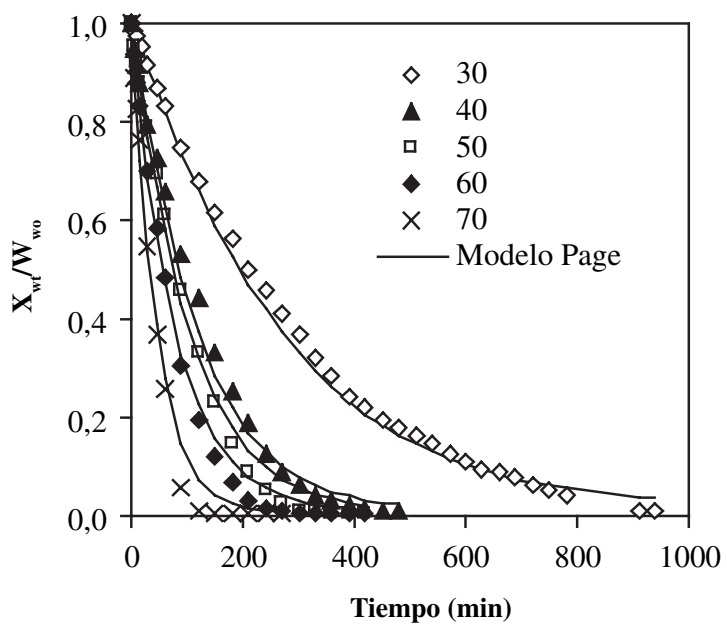

Figura 9. Curva de secado experimental y calculado con el modelo de Page para las diferentes temperaturas de secado $\left({ }^{\circ} \mathrm{C}\right)$. 


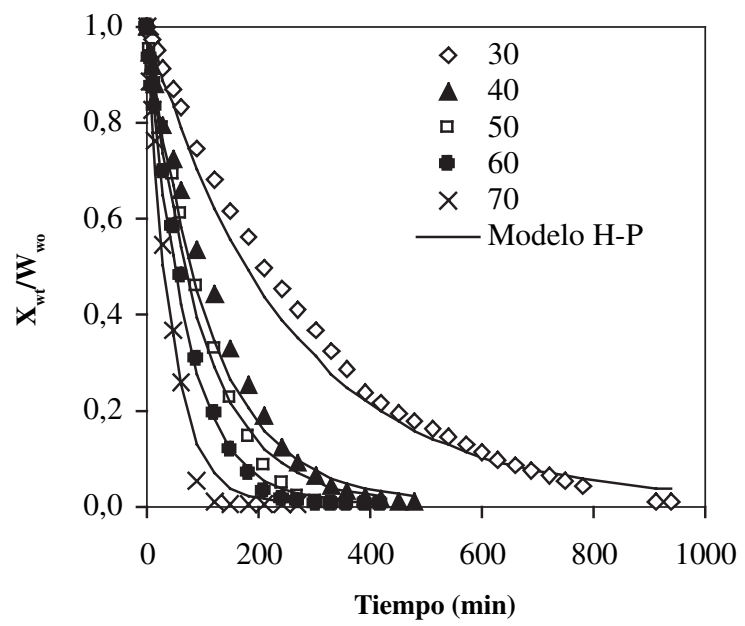

Figura 10. Curva de secado experimental y calculado con el modelo de Henderson-Pabis para las diferentes temperaturas de secado $\left({ }^{\circ} \mathrm{C}\right)$.

Al comparar los tres modelos empíricos utilizados, todos presentaron valores del coeficiente de regresión $\left(\mathrm{r}^{2}\right)$ superiores a 0.98 . No obstante, una vez analizados los otros estadísticos (SSE, RMSE y $\chi^{2}$ ) se comprobó que el modelo Page obtuvo la mejor calidad de ajuste sobre los datos experimentales (tabla 6) con respecto a las ecuaciones propuestas por Newton y HendersonPabis. La buena simulación de los datos experimentales que posee el modelo de Page se puede deber a que posee el parámetro exponencial $n$, el cual otorga una mayor aproximación matemática sobre las curvas de secado con tendencia exponencial [19, 20 y 27].

\section{CONCLUSIONES}

De acuerdo a los resultados obtenidos en esta investigación, se comprobó la validez de la ecuación de GAB para ajustar y modelar la isoterma de desorción de la Gracilaria chilena para las tres temperaturas de trabajo $\left(5,25\right.$ y $\left.40^{\circ} \mathrm{C}\right)$.

Se observó una marcada influencia de la temperatura sobre la cinética de secado por aire caliente de este producto y en general los tres modelos propuestos para simular el proceso de secado (Newton, Henderson-Pabis y Page) mostraron una buena calidad de ajuste sobre las curvas de secado experimentales, validando su utilización.

Los valores de la difusividad $\left(\mathrm{D}_{\text {we }}\right)$ encontrados estuvieron dentro del rango de 2.76 a $22.41 \times 10^{-9} \mathrm{~m}^{2} \cdot \mathrm{s}^{-1} \mathrm{y}$ dependen directamente de temperatura de secado, permitiendo calcular una energía de activación de $39.92 \mathrm{~kJ} \cdot \mathrm{mol}^{-1}$.
Al evaluar esta dependencia con la temperatura aplicando Arrhenius se obtuvo una energía de activación para las constantes cinéticas $\mathrm{k}_{1}, \mathrm{k}_{2} \mathrm{y} \mathrm{k}_{3}$ de 33.85, 33.49 y 33.83 $\mathrm{kJ} \cdot \mathrm{mol}^{-1}$, respectivamente. Sin embargo, los parámetros $a$ y $n$ permanecieron constantes con la temperatura.

Por último, de acuerdo a los resultados estadísticos aplicados, el modelo de Page fue el que presentó la mejor calidad de ajuste de los datos experimentales y representa una buena herramienta para el cálculo del tiempo de secado de la Gracilaria Chilena.

\section{NOMENCLATURA UTILIZADA}

$\mathrm{a}_{\mathrm{w}}=$ actividad de agua (adimensional)

$\% \mathrm{HR}=$ humedad relativa $(\%)$

$\mathrm{X}_{\mathrm{wt}}=$ humedad de la muestra (g agua/g m.s.)

$\mathrm{X}_{\text {wo }}=$ humedad inicial (g agua/g m.s.)

$X_{w e}=$ humedad en equilibrio (g agua/g m.s.)

$\mathrm{X}_{\mathrm{m}}=$ humedad de la monocapa (g agua/g m.s.)

$\mathrm{C}, k=$ parámetros de la ecuación de GAB

$\mathrm{N}=$ cantidad de datos

$\mathrm{Z}=$ número de constantes

$\mathrm{D}_{\mathrm{we}}=$ coeficiente difusional efectivo de agua $\left(\mathrm{m}^{2} \cdot \mathrm{s}^{-1}\right)$

$\mathrm{t}=$ tiempo de secado (min.)

$\mathrm{MR}=$ relación de humedad (adimensional)

$\mathrm{MRe} i=$ relación de humedad experimental (adimensional)

$\mathrm{MR} c i=$ relación de humedad calculada (adimensional)

$\mathrm{k}_{1}=$ parámetro del modelo de Newton

$\mathrm{k}_{2}, n=$ parámetros del modelo de Page

$\mathrm{k}_{3}, a=$ parámetros del modelo de Henderson-Pabis

$\mathrm{i}=$ número de términos

\section{AGRADECIMIENTOS}

Los autores agradecen a la Dirección de Investigación de la Universidad de La Serena (DIUILS) por el financiamiento otorgado para esta investigación.

\section{REFERENCIAS}

[1] I. Gómez, F. Figueroa, P. Huovinen, N. Ulloa, V. Morales. "Photosynthesis of the red alga Gracilaria chilensis under natural solar radiation in an estuary in southern Chile". Aquaculture. Vol. 224. No 1-4, pp. 369-382. 2005.

[2] Sernapesca. Servicio Nacional de Pesca, Chile. Visitada en mayo de 2004. Disponible en: http://www.sernapesca.cl 
[3] IFOP. Instituto de Fomento Pesquero, Chile. Visitada en mayo de 2004. Disponible en: http://www. ifop.cl

[4] L.A. Mohamed, M. Kouhila, S. Lahsasni, A. Jamali, A. Idliman, M Rhazi, M. Aghfir, M. Mahrouz. "Equilibrium moisture content and heat of sorption of Gelidium sesquipedale". Journal of Stored Products Research. Vol. $41 \mathrm{~N}^{\mathrm{o}} 2$, pp. 199-209. 2005.

[5] D. Barbanti, D. Mastrocola, C. Severini. "Air drying of plums: a comparison among twelve cultivars". Sciences des Aliments. Vol. $14 \mathrm{~N}^{0} 1$, pp. 61-73. 1994.

[6] I. Doymaz. "Drying kinetics of white mulberry". Journal of Food Engineering. Vol. $61 \mathrm{~N}^{\mathrm{o}} 3$, pp. 341-346. 2004.

[7] M.K. Krokida, V.T. Karanthanos, Z.B. Maroulis, D. Marinos-Kouris. "Drying kinetics of some vegetables". Journal of Food Engineering. Vol. $59 \mathrm{~N}^{\text {o }}$ 4, pp. 391-403. 2003.

[8] E.K. Akpinar. "Determination of suitable thin layer drying curve model for some vegetables and fruits". Journal of Food Engineering. Vol. $73 \mathrm{~N}^{\mathrm{o}}$ 1, pp. 75-84. 2005.

[9] F. Kaymak-Ertekin. "Drying and rehydration kinetics of green and red peppers. Journal of Food Science. Vol. $67 \mathrm{~N}^{\mathrm{o}}$ 1, pp. 168-175. 2002.

[10] S. Simal, A. Femenia, M.C. Garau, C. Roselló. "Use of exponential, Page's and difusional models to simulate the drying kinetics of kiwi fruits". Journal of Food Engineering. Vol. $66 \mathrm{~N}^{\mathrm{o}} 3$, pp. 323-328. 2005.

[11] A. Vega y R. Lemus. "Modelado de la cinética de secado de la papaya chilena (Vasconcellea pubescens)". Revista Información Tecnológica. Vol. $27 \mathrm{~N}^{\circ}$ 3, pp. 23-31. 2006.

[12] A.O.A.C. "Official Method of Analysis". Association of Official Analytical Chemists. N 934.06. Arlington, USA. 1990.

[13] W. Spiess and W. Wolf. "The results of the COST 90 project on water activity". In Physical Properties of Foods". Eds R. Jowitt et al. Applied Science Publisher. London, pp. 65. 1983.

[14] A. Vega, A. Andrés y P. Fito. "Modelado de la cinética de secado del pimiento rojo (Capsicum
Annuum L.) cv Lamuyo. Revista Información Tecnológica. Vol. $16 \mathrm{~N}^{\mathrm{o}}$ 6, pp. 3-11. 2005.

[15] E.O. Timmermann, J. Chirife and H.A. Iglesias. "Water sorption isotherm of foods and foodstuffs: BET or GAB parameters?". Journal of Food Engineering. Vol. $48 \mathrm{~N}^{\mathrm{o}}$ 1, pp. 19-31. 2001.

[16] J. Crank. "The mathematical of diffusion". 2nd Ed. London. Oxford University Press. 1975.

[17] I.T. Toğrul and D. Pehlivan. "Modelling of drying kinetics of single apricot". Journal of Food Engineering. Vol. $58 \mathrm{~N}^{\mathrm{o}}$ 1, pp. 23-32. 2003.

[18] S.J. Babalis and V.G. Belessiotis. "Influence of the drying conditions on the drying constants and moisture diffusivity during the thin-layer drying of figs". Journal of Food Engineering. Vol. 65 No 3, pp. 449-458. 2004.

[19] I. Doymaz. "Drying characteristics and kinetics of okra". Journal of Food Engineering. Vol. 69 $\mathrm{N}^{\mathrm{o}}$ 3, pp. 275-279. 2005.

[20] G. Mwithiga and J.O. Olwal. "The drying kinetics of kale (Brassica oleracea) in a convective hot air dryer". Journal of Food Engineering. Vol. 71 $\mathrm{N}^{\mathrm{o}}$ 4, pp. 373-378. 2005.

[21] I. Doymaz. "Thin-layer drying behavior of mint leaves". Journal of Food Engineering. Vol. 74 No 3, pp. 370-375. 2006.

[22] S.S.H. Rizvi. "Thermodynamic properties of food in dehydration". In M.A. Rao. and S.S.H. Rizvi (Eds). Engineering Properties of Foods. 2nd Edition Revised and Expanded. New York Marcel Dekker, Inc. pp. 223-309. 1995.

[23] P.C. Panchariya, D. Popovic and A.L. Sharma. "Thin-layer modeling of black tea drying process". Journal of Food Engineering. Vol. $52 \mathrm{~N}^{\mathrm{o}} 4$, pp. 349-357. 2002.

[24] W. Senadeera, B.R. Bhandari, G. Young and B. Wijesinghe. "Influence of shapes of selected vegetable material son drying kinetics during fluidized bed drying. Journal of Food Engineering. Vol. $58 \mathrm{~N}^{\mathrm{o}} 3$, pp. 277-283. 2003.

[25] V.T. Karathanos and V.G. Belessiotis. "Application of a thin-layer equation to drying data of fresh and semi-dried fruits". Journal of Agricultural Engineering Research. Vol. 74 No 4, pp. 335-361. 1999. 
[26] D. Velić, M. Planinić, S. Tomas and M. Bilis. "Influence of airflow velocity on kinetics of convection apple drying". Journal of Food Engineering. Vol. $64 \mathrm{~N}^{\circ}$ 1, pp. 97-102. 2004.
[27] S. Azzouz, A. Guizani, W. Jomaa and A. Belghith. "Moisture diffusivity and drying kinetic equation of convective drying of grapes". Journal of Food Engineering. Vol. $55 \mathrm{~N}^{\mathrm{o}}$ 4, pp. 323-330. 2002. 